\title{
IoT based Wearable Safety Device for Women
}

\author{
${ }^{1}$ Dhruvil Parikh, ${ }^{1}$ Pallavi Kapoor, \\ ${ }^{1}$ Shital Karnani \\ ${ }^{1}$ Student \\ Dept. of E\&Tc, Bharati Vidyapeeth \\ (Deemed to be University), College of Engineering, \\ Pune, India
}

\author{
${ }^{2}$ Prof. Sudhir Kadam \\ ${ }^{2}$ Professor \\ Dept. of E\&Tc, Bharati Vidyapeeth \\ (Deemed to be University), College of Engineering, \\ Pune, India
}

\begin{abstract}
The paper presents a Wearable safety device for women using the NodeMCU microcontroller. The research purpose of this device is to safeguard women in the event they might face any danger. The device uses IoT to communicate with the secure channels and to send alerts to them. The device is programmed in such a way that as soon as the sensor readings go above the threshold values the distress algorithm is initiated. The GPS and GSM are used to ping the user's location directly to the relevant authorities and saved contacts. The two additional switches in the device work for sending manual alerts in case of emergency and disengaging the system in case of generation of the false alerts respectively. ThingSpeak is an IoT platform that allows us to connect and save sensor data in the cloud and develop IoT applications. The advantage of this platform helps us in uploading the data on ThingSpeak after every 30sec so that continuous health monitoring of the individual can also be done. Taking into account this, the previously mentioned framework is a wellspring of building up an item for people which helps in keeping up the security of the user by taking out the inclusion of users to start any procedural exercises against any circumstance which the user might feel at serious risk.
\end{abstract}

Keywords—IoT, ThinkSpeak, Women Safety, GSR, NodeMCU, MAX30102

\section{INTRODUCTION}

In this twentieth-century where everyone is allowed to do whatever they want and to roam wherever they wish to go. There is a section or say half the section of the society who are still constrained to live their lives to the fullest. Yes, we are talking about females. Women are still suffering from various inequalities. According to the National Crime Records Bureau, in 2015 , there were over 300,000 reported incidents, a $44 \%$ increase from 2011 crimes against women. They are the foundation of any economy essentially forming the eventual fate of the nation. She who earlier stayed at home to attend her domestic duties is now maintaining work and home simultaneously, participating in the process of economic development on an equal footing with men.

So, it is the time to think about their safety and it is possible through IoT (Internet of Things). The main answer for the issue can be taken so that the women ought to be allocated with a well-being device that is convenient and guarantees her security. Our task centers around giving a Smart contraption dependent on IoT arrangements that not just serve to female get away from the basic circumstances but also additionally guarantees to give equality to the women.

IoT is the colossal number of interconnected gadgets over the web fit for overseeing computerized undertakings adroitly with no direct human inclusion. As time is passing, an ever- increasing number of gadgets are being interconnected and IoT is turning into an indispensable piece of our lives which permits us to play out the day by day undertakings quicker and proficiently, and in manners, we couldn't previously. In a perfect world, IoT will upgrade the future schedules with canny and solid frameworks that will make our way of life calm and furthermore adjust to the ever-developing needs. It will empower us to have totally robotized frameworks that will have its applications in making our security courses of action astute, by evacuating the client obstruction with any security gadget. More or less, IoT has the ability to meet all our needs before we even acknowledge what we need and will require. Security and interconnectedness are the genuine powers of IoT arrangements. Wearable security gear is another area that needs more research to have such progressive services to be fitted in ordinary wear however the arrangement may exist in IoT.

This is made possible by our device which not only is equipped with all the safety requirements but also sends the messages to the pre-assigned contacts in case of a dangerous situation. The main point of this work is to build up a wearable gadget for the security of women. This goal is accomplished by the examination of physiological signals related to body reaction parameters. The physiological signs that are investigated are galvanic skin reaction, body heartbeat, and body vibrations. This gadget is customized to ceaselessly screen the subject's parameters and make a move when any perilous circumstances introduce itself. The body vitals of an individual in dangerous situation changes and when these vitals cross the ideal values a distress algorithm gets initiated. The location of an individual is sent to the desired contacts to get the nearest help possible autonomously. The data is sent to the cloud for the regular health monitoring of an individual. Tactile switches are the additional feature in the gadget which makes it more efficient. The safety device will act as a guardian to the women at all times.

\section{LITERATURE SURVEY}

The following survey helped us in finding the right set of sensors and modules for building our proposed model.

[1] This paper suggests a security system that can ensure the safety of women and provide the necessary security measures. In this paper they suggested the use of various modules like GSM shield (SIM900A), Atmega328 board, 
Arduino Board, GPS(GYGPS6MV2) module, Screaming alarm (ADR 9600), Pressure sensor and power supply unit.

[2] This paper portrays wearable sensor nodes with solar energy harvesting. This paper contains records about distinct sensors that are applied for the checking of the health data of an individual. Additionally, they've created one online application to monitor the sensor's node information.

[3] In this paper they are building one wearable health monitoring system which is valuable to check the health of patients. They are giving data about sensors and the working range of sensors and Bluetooth.

[4] This paper proposes one Smart Watch with GPS. Also, this system contains an electric shock generator module, a screaming alarm module, a voice recognition module that is useful for women's security. The smart band contains three sensors that are a temperature sensor, pulse rate sensor, and motion sensor. The system can perform real-time monitoring and detect the situation of women in a critical situation. This system can detect the location and health condition of a person that will help to take action accordingly.

\section{SYSTEM OVERVIEW}

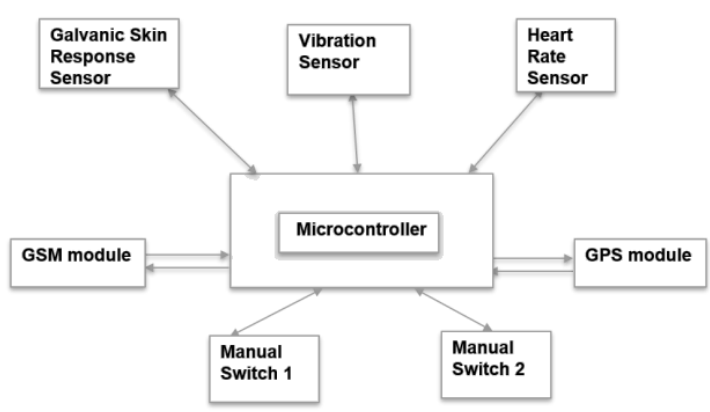

Fig. 1. Block Diagram of the System

The proposed system in the present project work is portrayed in Fig. 1.1 shown above in a highly remote manner with various blocks numbered which are explained as follows:

Block 1 gives knowledge about Galvanic Skin Response Sensor. The galvanic skin response (GSR), also named Electrodermal Activity (EDA) and Skin Conductance (SC) is the measure of the unceasing variations in the electrical attributes of the skin, caused by the variation of the human body sweating.

Block 2 gives the knowledge about Vibration Sensor (SW420 module). Vibration Sensors are sensors for calculating, displaying, and studying linear velocity, displacement, and proximity, or acceleration. Therefore, vibration analysis is used as a tool to determine apparatus condition as well as the distinct location and type of complications.
Block 3 gives information about Pulse Rate Sensor (MAX30102). Pulse rate Sensor is a well-created plug-andplay heart-rate sensor for Arduino. This Sensor determines the pulse rate of the person.

Block 4 is the GPS unit. A GPS navigation system is a GPS receiver and audio/video (AV) peripheral constructed for a distinct goal such as a car-based or hand-held device or a smartphone app. The global positioning system (GPS) is a 24satellite navigation tool that uses various satellite signals to find a receiver's spot-on earth.

Block 5 is the GSM unit. SIM800L module is a GSM-GPS two in one function module. In our system we are using it to send the location of the victim along with the message to the contacts which are registered.

Block 6 is the manual switch 1 . This tactile switch is pressed manually by the user in case of any emergency.

Block 7 is the manual switch 2. This tactile switch is used by the user to instruct the beneficiary that the message which was sent previously was inaccurate and they have to just neglect the message

The microcontroller used is NodeMCU. NodeMCU is an open-source IoT platform. It consists of peripheral which runs on the ESP8266 Wi-Fi SoC from Espressif Systems and hardware which is based on the ESP-12 module. The word "NodeMCU" by default refers to the peripheral rather than the development kits.

\section{WORKING}

The complete working of the proposed system can be divided into different modules which are as follows:

a) Database Module: - The user requires to provide the emergency contact numbers into the system which will be saved in the code. The code used will also save the messages which should be sent in different situations.

b) Sensor Module: - The system separately applies the distress algorithm if the sensor module develops values greater than the threshold. As soon as the algorithm is applied system delivers the message along with the user location to the saved contacts. At the receiver just by ticking on the location link given in the message it can display the location on the Google map in terms of latitude and longitude.

c) Global Positioning System (GPS) module: - It is navigation and accurate positioning tool. It records the location in the form of longitude and latitude.

d) GSM System Module: - Global System for Mobile communication (GSM) SIM card is added inside the mobile device to deliver and obtain the messages using GPRS. 
e) Switching Modules: - Switches are used in the system in order to overcome the emergency. Manual Switch 1 is used in case of an emergency situation in which the person needs help. By clicking this his/her location will be automatically delivered to the saved contacts so that necessary action may be taken. This is the manual alert system. On the other hand, Manual switch, 2 is used to disassociate the system in case of incorrect initiation of the message.

f) ThingSpeak: - ThingSpeak is an IoT platform that saves sensor data in the cloud and develops IoT applications. In our project we are transmitting the data on ThingSpeak after each $30 \mathrm{sec}$ so that regular health monitoring of the person can also be done.

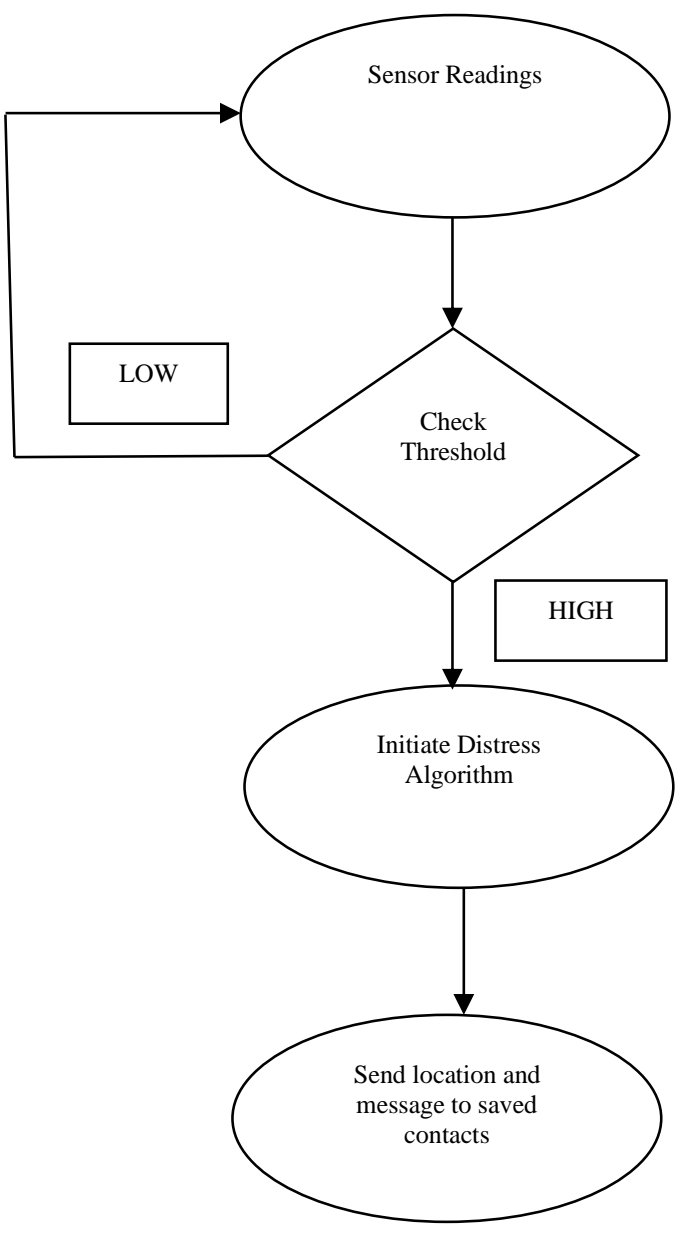

Fig. 2. Flow Chart of the System

The flowchart of the proposed system is shown in Fig. 2. The flowchart starts with continuously taking the readings from the sensors. The three Sensors will measure the body vitals continuously. These readings will be processed through a set of conditions. If the conditions are met or the recorded values will beyond the threshold value i.e. the normal ranges of the different sensors then the device will get triggered and the distress algorithm will get initiated. This will proceed with sending the location link in the form of latitude and longitude along with the appropriate message to the registered emergency contact numbers.

\section{RESULT ANALYSIS}

The proposed smart automated system provides a fully autonomous Security device that is tested under different cases to obtain better results. Some real-time scenario was created where the user might feel insecure to see the efficiency of the system. These scenarios can be easily differentiated from any normal stress-causing activity using the disabling tactile switch; at the same time emergency switch can be used to make the system forcefully work. Below are some pictures associated with the system.

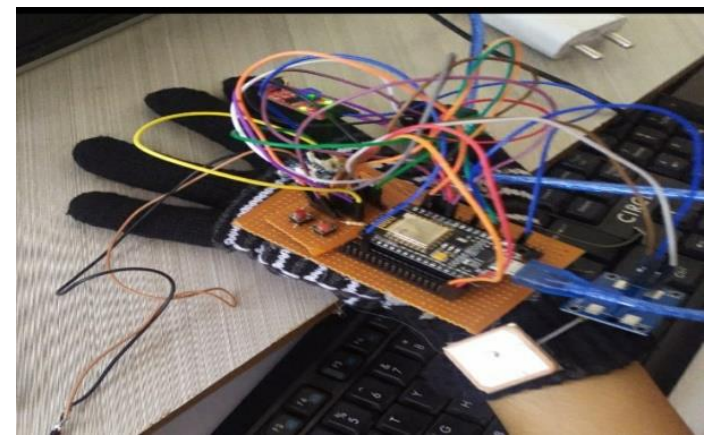

Fig. 3. Prototype

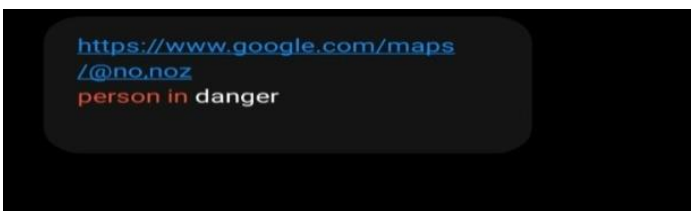

Fig. 4. When the Sensors' reading will cross the threshold value, this message along with the location link will be sent to the registered contacts.

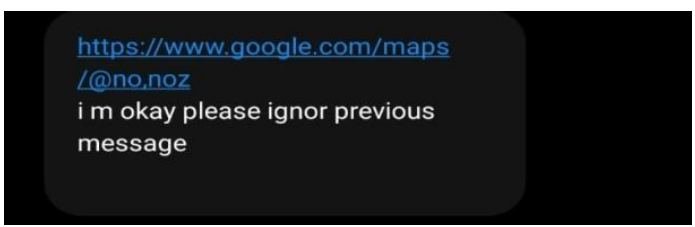

Fig. 5. If there is any false alarm, then this message along with the location link will be sent to the registered contacts.

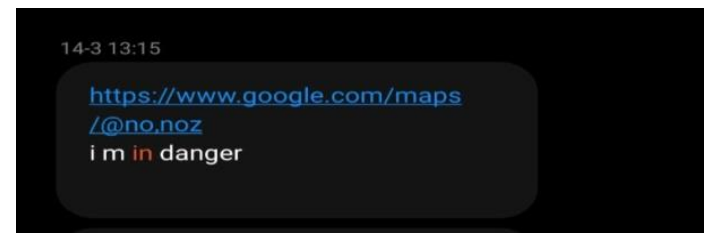

Fig. 6. For the emergency purpose, location link and this message will be sent to the saved contacts when the emergency switch is pressed

\section{FUTURE SCOPE}

With the advancement of technology and for better functionality of the product, new versions of the device can be introduced. The present system is efficiently working, but still to increase the functionality of the device, various other modules can be added without majorly affecting the present system. Among the various added functionalities, some could be voice detection, camera, screaming alarm, and shock generator 


\section{CONCLUSION}

With the world moving to the smarter lifestyle it has become an issue of prime importance to provide a secure system for women. The whole idea of this project is to provide a wearable safety device for women and at the same time can help in the regular health monitoring of an individual. The arrangements made to send the alert messages to the concerned authorities in case of an emergency proved to be working instantaneously with the different variations of inputs. The data of an individual is sent to the cloud for regular health updates. All the false alerts if triggered could be canceled with the help of the tactile switch and on the contrary, an emergency message could also be initiated in dangerous situations. The Internet of things has revolutionized the whole security system and this has resulted in a more compact and secure environment for the females.

\section{ACKNOWLEDGMENT}

The group members want to recognize the endeavors of our Guide, Prof. Sudhir Kadam. His persistent efforts have motivated us to accomplish our project. We want to thank our institute: Bharati Vidyapeeth (Deemed to be University), College of Engineering, for providing us with the opportunity to work on the project. Also, we want to express gratitude towards Mr. Avinash Magdum, Director, Whiz Key (OPC) Pvt. Ltd. for sponsoring our project.

\section{REFERENCES}

[1] Prof. Basavaraj Chougula, Archana Naik, Monika Monu, Priya Patil and Priyanka Das, "Smart girls security system," International Journal of Application or Innovation in Engineering \& Management (IJAIEM) ISSN:2319-4847 Volume 3, Issue 4, April 2014

[2] T. Wu, F. Wu, J. Redouté and M. R. Yuce, "An Autonomous Wireless Body Area Network Implementation Towards IoT Connected Healthcare Applications," in IEEE Access, vol. 5, pp. 11413-11422, 2017, doi: 10.1109/ACCESS.2017.2716344.

[3] K. Elissa, "Title of paper if known," unpublished. A. Pantelopoulos and N. G. Bourbakis, "A Survey on Wearable Sensor-Based Systems for Health Monitoring and Prognosis," in IEEE Transactions on Systems, Man, and Cybernetics, Part C (Applications and Reviews), vol. 40, no. 1, pp. 1-12, Jan. 2010, doi: 10.1109/TSMCC.2009.2032660.

[4] Shreyas R.S, Varun B.C, Shiva Kumar H.K, Punith Kumar B.E, Kalpavi C.Y, "Design and development of women self defence smart watch prototype," International Journal of Advanced Research in Electronics and Communication Engineering (IJARECE) ISSN: 2278 - 909X Volume 5, Issue 4, April 2016 